\title{
Research on conceptual design of mechatronic systems
}

\author{
XU YONG ${ }^{1}$, ZOU HUIJUN $^{1}$ and LI RUIQIN ${ }^{2}$ \\ ${ }^{1}$ School of Mechanical Engineering, Shanghai Jiaotong University, Huashan \\ Road, 1954, Shanghai, China \\ ${ }^{2}$ Department of Mechanical Engineering, North University of China, Xueyuan \\ Road 1, Taiyuan Shanxi, China \\ e-mail: brucexuyong@163.com
}

MS received 21 January 2006; revised 19 June 2006

\begin{abstract}
Based on the fact that function-structure generating and function solving are alternant processes with mutual causality during the conceptual design phase of mechatronic systems, a conceptual design cyclic feedback solving model of a mechatronic system is put forward on the basis of mapping between function layer, effect layer, working principle layer and structure layer. The process of solving single and system functions is analysed. Key technologies of interface matching and function solving are then advanced. Finally, a computer-aided conceptual design automatic software system for mechatronic systems is developed and the conceptual design of a computerised embroidery machine is given as an example.
\end{abstract}

Keywords. Conceptual design; interface matching; mechatronic system; cyclic feedback mapping.

\section{Introduction}

A mechatronic component or subsystem transfers and exchanges material, energy and information through interfaces. As combination boundary and function borderline, an interface reflects the degree of cohesion and coupling of mechatronic components. In a sense, mechatronic system design is fundamentally an 'interface design' (Jianmin et al 2001). The essence of interface matching among mechatronic components is to describe relations of interface variables which depict input-output characteristics and states of material, energy and information flow. Thus, research on matching of component interfaces is an indispensable base for mechatronic system design.

Conceptual design is the most innovative stage in product design process, which has decisive influence on improving product performance and reducing product costs. Conceptual design is a solving process from function to structure scheme (Bracewell \& Sharpe 1996; Qian \& Gero 1996; Feng \& Xu 1996; Min-de et al 1999; Nam 2001; Ruiqin 2004). At present,

This work was supported by the National Natural Science Foundation of China (Grant No. 50375045) and the Shanxi Natural Science Foundation of China (Grant No. 20041070) 
research on mechatronic integration and fusion is far from intensive. Due to inadequate treatment with coupling relations between mechanism, sensor and controller in the product, the holistic dynamic performance of mechatronic products is not satisfactory despite complete imitation of advanced products. So influences of matching and coupling of various subsystems/components on holistic dynamic performance of mechatronic systems must be taken into account from the initial conceptual design phase. Reasonable disciplinary integration and optimum holistic performance of mechatronic system should be achieved by harmony among subsystems/components.

This paper analyses the respective solving processes for single and system functions of mechatronic systems which mostly accomplish movement executive functions, and for the first time puts forward a conceptual design cyclic feedback solving model of such systems based on multilayer mapping, and advances such key technologies as interface matching and function solving. Computer-aided conceptual design of a computerised embroidery machine is given as an example.

\section{Process model for conceptual design of a mechatronic system}

\subsection{Single-function solving process of mechatronic system}

During single-function solving, effects that can realise a certain function are pondered first, and working principles which can realise a certain effect are sought afterwards. Finally, a structure scheme which can realize a certain working principle is searched for viz. the course of single-function solving proceeds by layer-by-layer mapping (FEPS for short) between function layer, effect layer, working principle layer and structure scheme layer in order.

Function explains realisation, the purpose of structure scheme solution, effect reflects the essence of structure scheme solution, working principle describes the basic mechanism for realising the function, and structure scheme is the embodiment of the foregoing three.

\subsection{System-function solving process of a mechatronic system}

The overall function of mechatronic system/product, herein termed system function, is realized by cooperation of multiple similar and/or different child functions. The core issue of function solving of mechatronic system comprises feasibility of each single-function solution and interface compatibility of various single-function solutions. System-function solving is continuance and evolution of single-function solving, while single-function solving is premise and basis of system-function solving. Therefore system-function solving bears necessarily some traits of the single-function solving process besides its own characteristics.

Function-solving process of mechatronic system is partitioned into three child processes in correspondence with three core problems of function-solving of mechatronic system: simplifying the child process solves function decomposition (into child functions) problem; embodying child process solves child-function solving problem - the key problem of singlefunction solving; integrating child process solves combination problem of child-function solutions.

\subsection{The feedback cyclic solving model for conceptual design of a mechatronic system}

Conceptual design is a typical ill-definition solving problem. Lacking sufficient information about product function at the initial stage, it is unpractical to obtain the complete function structure only by decomposition of abstract function layer. Due to limitations of cognition ability 


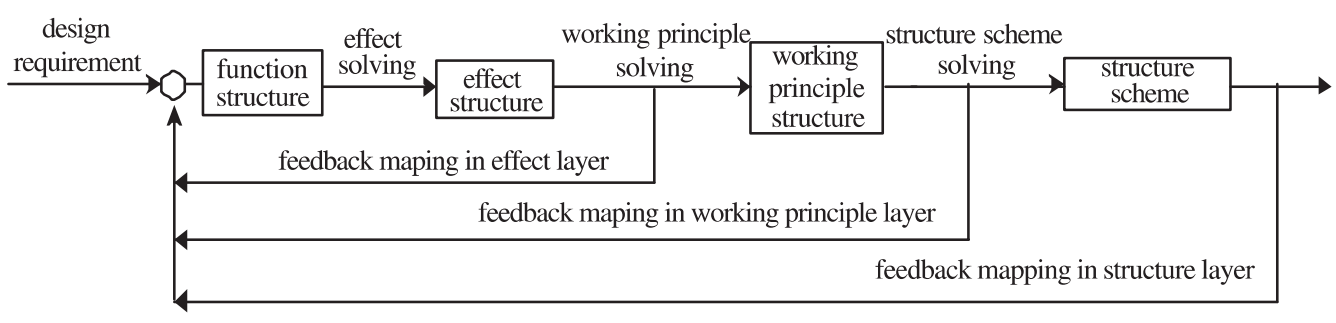

Figure 1. Cyclic feedback solving model for conceptual design of a mechatronic system.

and design experience in the designer, it is impossible to acquire immediately an ideal result for a serial function solving pattern. In fact, function structure generating and function solving are alternant processes with mutual causality. The differences between design requirements and existing design scheme can be eliminated only by the cyclic process of mapping solving and feedback iteration. Thus, a cyclic feedback solving model for conceptual design of mechatronic system is presented, as shown in figure 1 .

As shown in figure 1, the cyclic feedback solving model includes three mapping patterns, which correspond to three child processes of function solving of the mechatronic system. (1) Decomposing mapping pattern: This is actualized at the outset to reduce the degree of complexity of an existing complicated problem. Usually, function decomposing is done to yield function structure. (2) Solving mapping pattern: Layer-by-layer mapping pattern (FEPS) or inter-layer mapping pattern between function layer, effect layer, working principle layer and structure layer is implemented to gain effect solution, principle solution and structure scheme solution of the known child function. (3) Feedback mapping pattern: This is done to solve the problem of interface incompatibility between function solutions. When solutions of correlative child functions are combined to form the overall function solution, it is possible that corresponding interfaces conflict. In this case, an auxiliary child function has to be derived from the conflicting spot and then solved, before it is fed back to the previously mentioned function solution to eliminate known conflicts. In the foregoing mapping patterns, solving the mapping pattern is the core and principal part, while the decomposing mapping pattern prepares for its smooth progression and the feedback mapping pattern improves on its incomplete solutions. These three mapping patterns bear mutual causalities and advance alternately end to end. Together they constitute cyclic, closed loop feedback mapping in effect layer, working principle layer and structure layer, and finally generate reasonable and complete solutions of function structure, effect structure, working principle structure and structure scheme, as well as corresponding solutions of holistic structure scheme.

\section{Function solving of mechatronic system based on interface characteristic matching}

\subsection{Interface definition in a mechatronic system}

Function solving in a mechatronic system needs not only testing the feasibility of every single/child-function solution, but also matching the compatibility of various single-function solutions. So interface in mechatronic system is distinguished into two types: function-solving interface (SI for short) and function-solution combination interface (CI for short). SIs are interfaces which match one another during the function-solving process, and when SIs are incompatible, those corresponding solutions must be given up. CIs are interfaces which match 
one another during the combination of function solutions; when CIs are incompatible, these corresponding solutions need not be given up, but a new child function needs to be derived for interface transformation.

Corresponding to the four-layer structure of the cyclic feedback mapping model, interface characteristics of function solutions of a mechatronic system for conceptual design actually includes four levels, i.e., function level, effect level, principle level and structure level. Moreover, these levels are not coordinative; instead, the latter is an embodiment of the former. Thus there is a nested relation existing in connotation: the function interface characteristic is involved in the effect interface characteristic, the effect interface characteristic is involved in the principle interface characteristic, and the principle interface characteristic is involved in the structure scheme interface characteristic. The nested structure helps to solve feasibility and compatibility issues of design solutions concurrently. In this way, the interface characteristic defines in reality the interface constraints of function solutions for conceptual design at various levels. These interface constraints, applied mainly to search for feasible solutions in corresponding knowledge bases, are the kernel knowledge for function solving of system levels for conceptual design.

\subsection{Concurrent strategy of single-function solving and interface matching of a mechatronic} system

The premise and basis for function-solving of a mechatronic system is single-function solving. The interface compatibility inspection of single function solutions then proceeds seriatim along various layers according to correlation sequence. Incompatible function solutions in the previous layers should be abandoned in time in order to reduce interface inspection laboor in the next layer.

The overall function of a mechatronic system is commonly composed of six child functions, i.e., aim function, drive function, transmission function, sensing function, control function and auxiliary function (Ruiqin \& Huijun 2003). As movement executive function is usually the aim of a mechatronic system, the corresponding function solution is the kernel solution of the mechatronic system solutions and influences to a great extent or even decides on choices of the other child function solutions. So function solving can start from an elementary known movement executive function solution (usually aim function solution), first matched with a feasible drive function solution, and then pondered if it is necessary to select a transmission function solution between the movement executive function solution and the drive function solution. If necessary, a transmission function solution is required to be created. Its input interface must be compatible with that of the drive function solution and its output interface must be compatible with that of the movement executive function solution. The foregoing process is followed by matching appropriate sensing function solution, control function solution and finally auxiliary function solution, resulting in "concurrent strategy of single function solving and interface matching of mechatronic system' - a relatively effective and feasible matching strategy, as shown in figure 2.

\section{An application study}

Based on thoughts hereinbefore, a computer prototype system has been developed for conceptual design of the mechatronic system. Following the system sketch shown in figure 3, study of a computerized embroidery machine exemplifies the conceptual design process of such a system. 


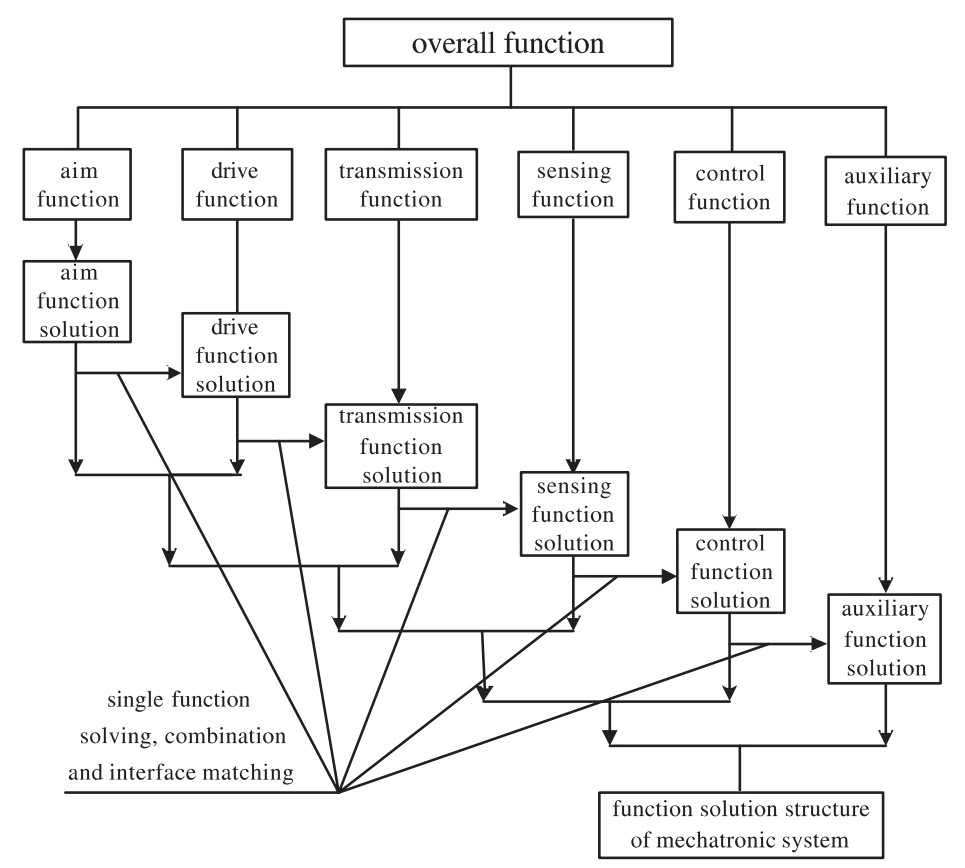

Figure 2. Concurrent strategy of single-function solving and interface matching of mechatronic system.

\subsection{A brief introduction to a computerized embroidery machine system}

A computerized embroidery machine is to be designed: embroidery patterns are stored in and outputted from the disk or EPROM by the embroidery plate-making system; the embroidery process principle is as follows: when the needle is above the fabric, the tabouret moves respectively in direction $x$ and $y$ to the prescribed position; when needle falls on the fabric surface from top down, the tabouret stops moving and the embroidering mechanism finishes a stitch. Moving distance of taboret respectively in direction $x$ and $y$ decides the length and the direction of a stitch. The aggregate of stitches composes embroidered fabric.

A computerized embroidery machine consists of such components as the motor, controller, sensor, tabouret transmission mechanism, embroidering mechanism, principal axis transmission mechanism etc. Owing to the limitation of space, here mainly studies solving problem of the movement executive function of tabouret in direction $x$.

\subsection{Aim function (movement executive function) reasoning and solving}

With the conflicting input and output interfaces as known conditions, the essence of function reasoning is a process of searching and matching respectively, with the input object interface and the output object interface in function knowledge base.

In this example, a function interface is first matched with an effect interface in the effect knowledge base. Obtained effects realizing the aim function of 'movement type conversion' are as follows: geometrical physics, lever, friction, wedge, polymeric force, circular movement, heat expansion, centrifugal force, bonding etc. The feasible effect thereinto is a geometrical physics effect, i.e. connections, positions and interaction relations of components by 


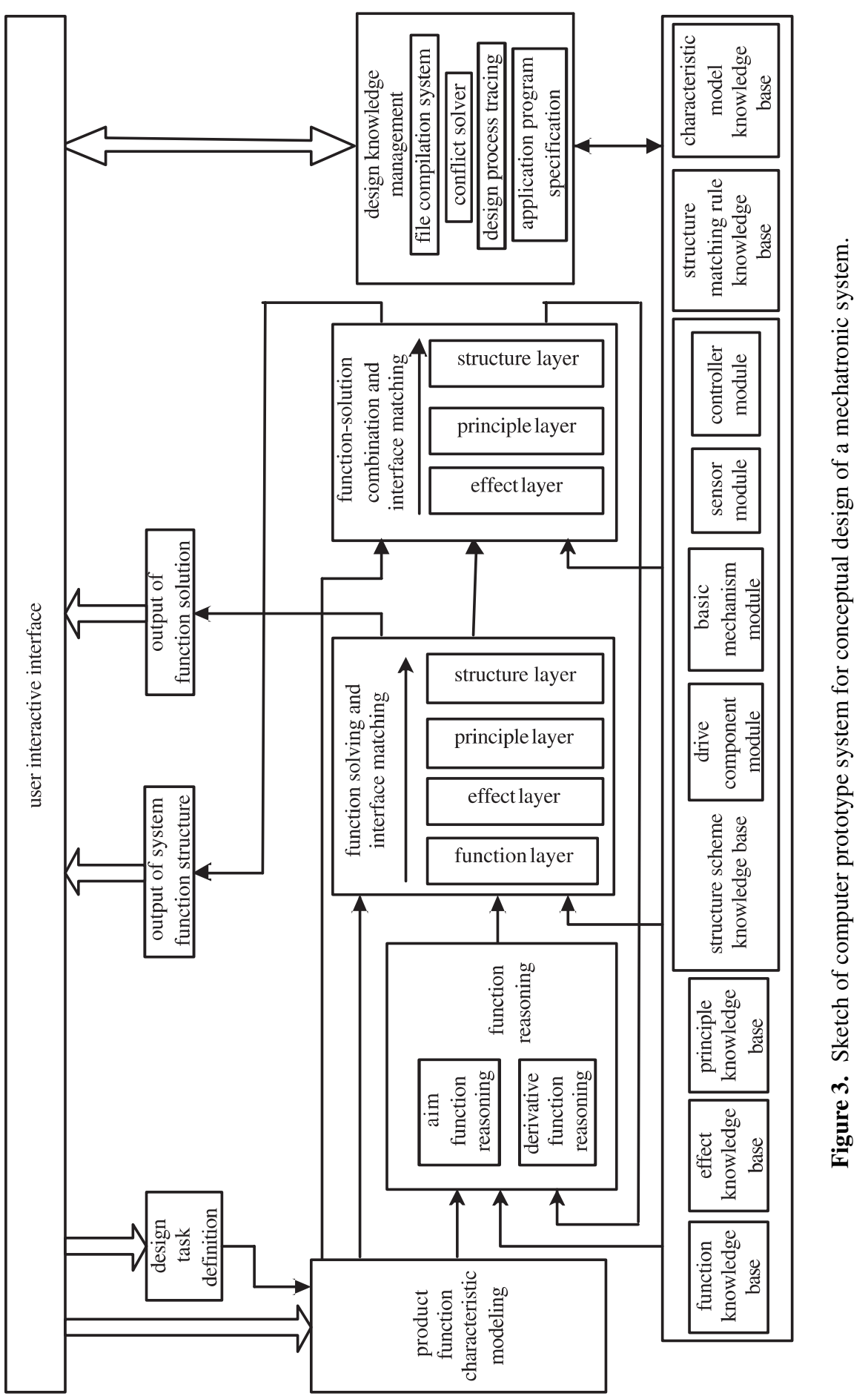




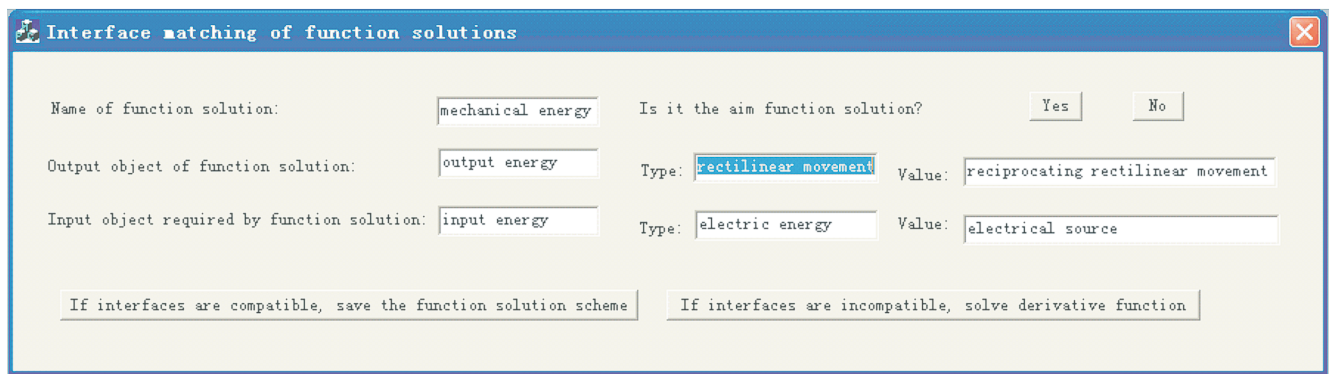

Figure 4. Compatibility judgment of aim function solutions.

kinematic pairs in a given space. Afterwards, working principle interfaces and structure interfaces are respectively matched in working principle knowledge base and structure scheme knowledge base to acquire corresponding working principle solutions and structure scheme solutions. The gained structure scheme solution realizing the movement executive function of the tabouret in this example is: tabouret motors rotate to drive the tabouret to move in the plane determined by axis $x$ and $y$.

\subsection{Compatibility judgment of aim function solutions and derivative function reasoning}

Combination compatibility of interfaces between two function solutions or between a solution and outer objects (action-object, environment and human) is examined layer-by-layer in the order of effect layer, working principle layer and structure layer. When conflict arises in the interface of structure scheme layer - the output energy required by the function solution is mechanical energy (reciprocating rectilinear movement in the plane), whereas the input energy of product function is electric energy, an accessorial single function needs to be derived to accomplish interface conversion from electric energy to mechanical energy.

Derivative function reasoning is applied at the spot where interfaces conflict. The known input and output interfaces are searched and matched respectively with the input object interface and the output object interface in the function knowledge base. The derivative single function obtained is the drive function (realizing conversion from electric energy to mechanical energy).

The picture for compatibility judgment of the aim function solution in the example is shown as figure 4. The input interface of a function solution is compared with its output interface, and the function solving process ends if the interfaces are compatible. The button at bottom left corner is pressed, and the computer system saves the scheme automatically. If function solution interfaces are incompatible, the button at bottom right corner is pressed, and the computer system proceeds automatically to the process of derivative function reasoning and solving.

\subsection{Drive function solving, compatibility judgment of solutions and derivative function rea- soning}

The processes of compatibility judgment of drive function solutions and derivative function reasoning are similar to those of the aim function above.

Drive function solving is as follows. With components available for selection, mapping solutions takes place directly from the function layer to the structure layer. Such drive solutions 
as stepper motor and alternating current servo motor are acquired through searching in the drive function knowledge base.

Compatibility judgment of solution is done by examining whether stepper motor interface matches the tabouret interface of movement function solution in direction $x$. We may see that interfaces in structure scheme layer are still incompatible: the output movement of tabouret transmission mechanism reciprocates rectilinear movement, whereas the output movement of stepper motor is circular. Therefore, an accessorial single function needs to be derived to accomplish relevant interface conversion from circular movement to reciprocating rectilinear movement.

Derivative function reasoning is as follows. The known input interface and output interface at the conflict spot are searched and matched respectively with the input object interface and the output object interface in function knowledge base. The single function obtained is the transmission function (realizing conversion from circular movement to reciprocating rectilinear movement).

\subsection{Solving and compatibility judgment of transmission function}

Carry out mapping solutions directly from function layers to structure layers. Tabouret transmission mechanism solutions such as synchronous cog belt, gear-rack mechanism and cam-linkage combination mechanism can be acquired by searching in transmission function knowledge base.

A picture of the principal interface of knowledge base for basic movement function structures is shown in figure 5 . The actual search proceeds as follows.

(1) Primary searching conditions are set, with 'circular movement' as the input movement, and 'reciprocating rectilinear movement' as the output movement; (2) secondary searching condition is set with 'working distance augmentation' as the attribute item, thereby five mechanism schemes are obtained; (3) according to the 'principle, characteristic and application'

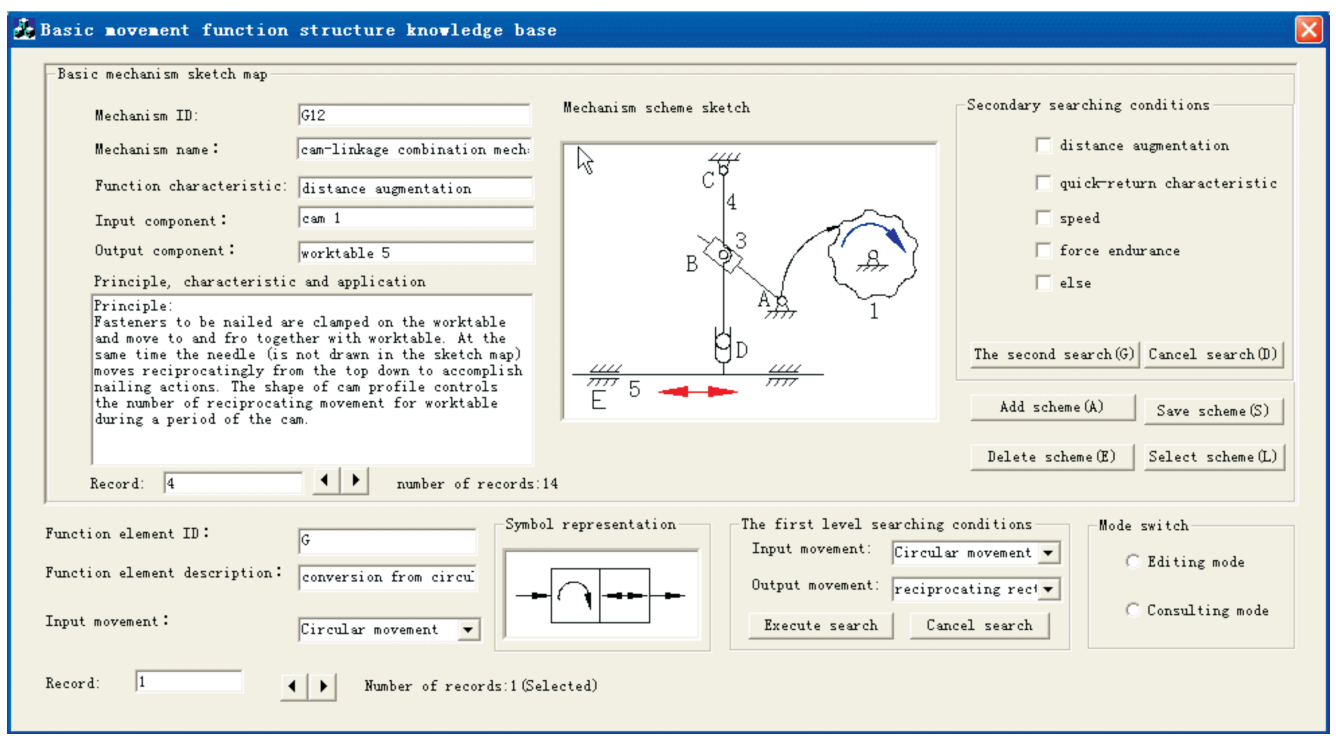

Figure 5. A reciprocating rectilinear movement mechanism with long working distance. 
item and practical requirements, an appropriate scheme thereinto can be selected and innovative schemes may be generated by analogy. Figure 5 shows a reciprocating rectilinear movement mechanism with long working distance, viz. a cam-linkage combination mechanism is adopted as tabouret mechanism to achieve the conversion from circular movement of stepper motor axis to reciprocating rectilinear movement of tabouret in direction $x$.

Judgment of solution compatibility: as all interfaces are compatible, the conceptual design process ends.

\section{Conclusions}

It is proved that the presented conceptual design feedback cyclic solving model and layerby-layer matching strategy of interface characteristics are capable of solving judgments of solution feasibility and interface compatibility during the conversion process from single function solving to system function solving, which can help a designer to search and discover speedily manifold solutions during conceptual design stage for mechatronic systems. Study of a computerized embroidery machine exemplifies the validity of an automatic prototype system in the conceptual design of a mechatronic system.

The authors gratefully acknowledge the support provided by the National Natural Science Foundation of China and the Shanxi Natural Science Foundation of China (see footnote on p. 661).

\section{References}

Bracewell R H, Sharpe J E E 1996 Functional descriptions used in computer support for qualitative scheme generation - Schemebuilder. AI EDAM 10: 333-346

Feng P E, Xu G R 1996 Feature modeling based on design catalogues for principle conceptual design. AI EDAM 10: $347-354$

Li R 2004 Research on theory and method of scheme creative design of mechatronic system. School of Mechanical Engineering, Shanghai Jiao Tong University, Shanghai, China

Li R, Zou H 2003 Research situation and development prospect of theory on conceptual design of mechatronic products. Mach. Des. Res. 19(3):10-13

Nam P S 2001 Axiomatic design: Advances and applications (New York: Oxford University Press)

Pahl G, Beitz W 1992 Engineering design (Beijing: Mech. Ind. Press)

Qian L, Gero J S 1996 Function-behavior-structure path and their role in analogy-based design. AI EDAM 10: 289-312

Shen M, Feng P, Song Y 1999 Construction of mechanics effects based knowledge bank used in conceptual design for mechanical transmission system. Konstruktion (2): 11-16

Yu R, Shi J 2000 Theory and technology of artificial intelligence (Hangzhou: Zhejiang University Press)

Zhang J, Tang S, Feng S 2001 System design of mechanical and electronical integration (Beijing: Higher Education Press) 